\title{
Long-Term Effects of Blast Exposure: A Functional Study in Rats Using an Advanced Blast Simulator
}

\author{
Peethambaran Arun, Donna M. Wilder, Ondine Eken, Rodrigo Urioste, Andrew Batuure, Sujith Sajja, \\ Stephen Van Albert, Ying Wang, Irene D. Gist, and Joseph B. Long
}

\begin{abstract}
Anecdotal observations of blast victims indicate that significant neuropathological and neurobehavioral defects may develop at later stages of life. To pre-clinically model this phenomenon, we have examined neurobehavioral changes in rats up to 1 year after exposure to single and tightly coupled repeated blasts using an advanced blast simulator. Neurobehavioral changes were monitored at acute, sub-acute, and chronic time-points using Morris water maze test of spatial learning and memory, novel object recognition test of short-term memory, open field exploratory activity as a test of anxiety/depression, a rotating pole test for vestibulomotor function, and a rotarod balance test for motor coordination. Single and repeated blasts resulted in significant functional deficits at both acute and chronic time-points. In most functional tests, rats exposed to repeated blasts performed more poorly than rats exposed to single blast. Interestingly, several functional deficits post-blast were most pronounced at 6 months and beyond. Significant neuromotor impairments occurred at early stages after blast exposure and the severity increased with repeated exposures. The novel object recognition testing revealed short-term memory deficits at 6 and 12 months post-blast. The water maze test revealed impairments at acute and chronic stages after blast exposure. The most substantial changes in the blast-exposed rats were observed with the center time and margin time legacies in the open field exploration test at 6, 9, and 12 months post-blast. Notably, these two outcome measures were minimally altered acutely, recovered during sub-acute stages, and were markedly affected during the chronic stages after blast exposures and may implicate development of chronic anxiety and depressive-like behaviors.
\end{abstract}

Keywords: advanced blast simulator; blast exposure; brain injury; chronic effects; neurobehavioral functions

\section{Introduction}

B LAST-INDUCED TRAUMATIC BRAIN INJURY (BTBI) is widely accepted as the signature injury of the recent Iraq and Afghanistan wars and is increasingly a significant concern in the civilian population due to the increased use of improvised explosive devices in terrorist attacks. Clinical reports have indicated that $80 \%$ of the brain injuries in service members with mild TBI are related to blast exposure. ${ }^{1}$ The brain injury after blast exposure is multifaceted and is induced by different components of the blast explosion. The major defined mechanisms underlying blast injury are primary (overpressure/shockwaves injury), secondary (shrapnel injury), tertiary (acceleration/body impact injury), or quaternary (heat and toxic chemical injury) injuries. ${ }^{2}$ Among these four components, the primary injury caused by exposure to blast overpressure is least understood and most common in victims with mild
TBI (mTBI). Thus, most of the pre-clinical studies of bTBI are focused on the effects of exposure to the primary injury component, blast overpressure.

Blast exposure appears to cause both acute and delayed neurobehavioral abnormalities and the severity of these injuries increases with blast intensity (overpressure) and number of blast exposures. The effects of mild TBI include symptoms such as headache, dizziness, and fatigue, fogginess, as well as impairments in cognitive, vestibular, oculomotor, and psychological functions, ${ }^{3-6}$ which may evolve into chronic symptoms in 15 to $30 \%$ of the cases. ${ }^{4,7}$ Many of the chronic symptoms of mTBI overlap with those of posttraumatic stress disorder (PTSD), which are reported in up to $40 \%$ of service members who have had mTBI. ${ }^{8}$ Combat-related mTBI often is associated with chronic neurocognitive impairments and symptoms of PTSD which are exacerbated by previous exposures to combat-related blast. ${ }^{9}$ Additionally, blast exposure also has been

Blast-Induced Neurotrauma Branch, Center for Military Psychiatry and Neuroscience, Walter Reed Army Institute of Research, Silver Spring, Maryland. 
reported to cause chronic white matter abnormalities, which are associated with long-term memory impairment. ${ }^{10,11}$

Pre-clinical studies applying primary blast exposure to experimental animal models also revealed acute and chronic neurobehavioral deficits after single and repeated blast exposures, which include neuromotor dysfunctions, short- and long-term memory defects, as well as anxiety and depression-like symptoms, especially at acute stages. ${ }^{12-21}$ Repeated exposure to low-level blasts generated in a constant diameter cylindrical shock tube resulted in acute and chronic anxiety- and depression-like symptoms in rats. ${ }^{22-24}$ In these studies, rats were exposed to daily 10.8 psi blast for 3 consecutive days and evaluated for anxiety-related and PTSDlike symptoms for up to 35 weeks post-blasts. ${ }^{22,23}$

Despite the recognition of blast TBI as the signature injury of recent wars and growing awareness of its association with persistent behavioral perturbations, few if any pre-clinical studies have been systematically carried out to longitudinally define the evolution of long-term neuropathological and neurobehavioral deficits occurring in experimental animal models after moderate or severe blast exposure. To our knowledge, the latest time-point used for assessments of neurobehavioral function in rats after moderatelevel blast exposure was 3 months post-blast, at which time shortterm memory loss and anxiety-like symptoms were observed. ${ }^{17}$ The severity of cognitive deficits at 6 or 12 months after moderate or severe blast exposures is largely unexplored, and would establish to what extent recovery or continued decline from acute blast-induced disruptions might occur.

In the present study, we have evaluated neurobehavioral functions in laboratory rats from 1 day to 1 year after single and tightly coupled repeated blast exposures using an Advanced Blast Simulator. For repeated blast exposures, as reported earlier, ${ }^{25}$ two tightly coupled exposures were used to simulate closely occurring blasts as can be encountered with improvised explosive device detonations as well as with the firing of high overpressure weapon systems, such as artillery.

\section{Methods}

\section{Animals}

All animal experiments were conducted at Walter Reed Army Institute of Research (an AAALAC International accredited research facility) in accordance with the Animal Welfare Act and other federal statutes and regulations relating to animals and experiments involving animals, and adhered to principles stated in the Guide for the Care and Use of Laboratory Animals (NRC Publication, 2011 edition) using an Institutional Animal Care and Use Committee approved protocol. Male Sprague Dawley rats 9-10 weeks old that weighed 300-350 g (Charles River Laboratories, Wilmington, MA) were housed at $20-22^{\circ} \mathrm{C}(12-\mathrm{h}$ light/dark cycle). Rats were given free access to nutritious rat chow (Prolab IsoPro RMH3000 from LabDiet, St. Louis, MO) and water ad libitum until 1 month after the blast exposure, when they reached a body weight of $400-450 \mathrm{~g}$. Since an increase in body weight above $500 \mathrm{~g}$ significantly affects performances in several of the neurobehavioral tests performed, diets for all rats were restricted after 1 month so that body weights were maintained between 450 and $500 \mathrm{~g}$ until the completion of the study (1 year). Body weights were recorded 3 days a week and adjustments were made in the quantity of diet to maintain body weights within this range.

\section{Primary blast exposure}

A critical aspect of pre-clinical evaluations of blast exposure is the appropriate simulation of blast in the laboratory, and as noted in a recent timely review, poorly designed blast simulations have confounded much of the pre-clinical biomedical blast literature to date. ${ }^{26}$ This includes a critical understanding of shockwave phenomena, the relationships among varied pressure recordings (e.g., static pressure, dynamic pressure, reflected pressure, and total or stagnation pressure) and how they relate to the loading conditions experienced by the experimental subject. To improve the ecological validity of our blast simulations, a state-of-the-art Advanced Blast Simulator (ABS) was used for these experiments (Fig. 1). With a divergent transition section and an end wave eliminator, this device eliminates the artifacts of commonly-used constant diameter (cylindrical) shock tubes (e.g., plateau or flat-top waveforms and exaggerated dynamic pressure [i.e., blast wind]) and provides a means to produce a controlled high-fidelity simulation of blast in the laboratory.

In contrast to the traditional cylindrical shock tubes used earlier for simulating blast overpressure in the laboratory, the ABS generates free field Friedlander waveforms quite comparable to field blasts using explosives. The ABS consists of a 0.5-ft long compression chamber that is separated from a 21-ft long transition/expansion test section by rupturable Valmex ${ }^{\circledR}$ membranes (Mehler Technologies, VA). The compression chamber was pressurized with air, causing the membranes to rupture at a pressure dependent upon the thickness of the specific membrane sheet(s) separating the two chambers, yielding a supersonic blast wave that impacts the experimental subject in the test section. The critical biomechanical loading to the experimental subject is determined from both the static and dynamic pressure of the blast wave, which are fully recorded by a combination of side-on and head-on piezoresistive pressure gauges (Endevco Corporation, CA) using an Astro-med TMX-18 acquisition system at an $800,000 \mathrm{~Hz}$ sampling rate. Similar ABSs have been recently used in reports from other laboratories for blast exposure, but these studies did not address long-term neurobehavioral functional assessments in rodents after blast exposure. $^{27,28}$

For blast exposure, rats were anesthetized with $4 \%$ isoflurane for 8 min and secured by nylon netting to an aluminum platform in a longitudinal (i.e., rat facing the oncoming shockwave) prone orientation in the test section $\left(2^{\prime} \times 2^{\prime}\right.$ inner dimensions). To produce moderate TBI in rats in these experiments, we used Valmax membranes (0.034 inches thick) yielding peak positive static pressures of approximately $19 \mathrm{psi}$ with a positive phase duration of 4-5 msec. For tightly coupled repeated blast exposures, the animals were exposed to two 19 psi blast overpressure waves separated by $2 \min$.

\section{Rotating pole test}

A rotating pole test was used to assess neurological motor dysfunction after bTBI. The device, described by Mattiasson and colleagues $^{29}$ for use in TBI, consists of a wooden pole, $4 \mathrm{ft}$ in length and 1.5 inches diameter, which is suspended parallel to the floor at a height of approximately $3 \mathrm{ft}$ overlying a foam cushion. The pole was connected to a variable speed motor, which in turn was connected to a variable direct current power supply. One end of the pole leads into a dark enclosed plastic box. All rats underwent training on the rotary pole prior to baseline (pre-blast) measurements.

Training consisted of repeatedly gently placing the rat on the pole at increasing distances from the box and clapping to encourage it to move to the box. The training was done 1 day before blast exposure and also 1 day before retesting the animals on Months 1, 3 , and 6 . On each test day, the rats were positioned at the opposite end of the rotating pole and were allowed to traverse the pole at their own speed and enter the box. Each rat was timed as it traversed the pole. Once it entered the plastic box, the entry was closed, and the rat allowed to remain in the seclusion box for $30 \mathrm{sec}$. During the testing, each rat had $120 \mathrm{sec}$ to complete each run. Rats were tested 


\section{Advanced Blast Simulator}

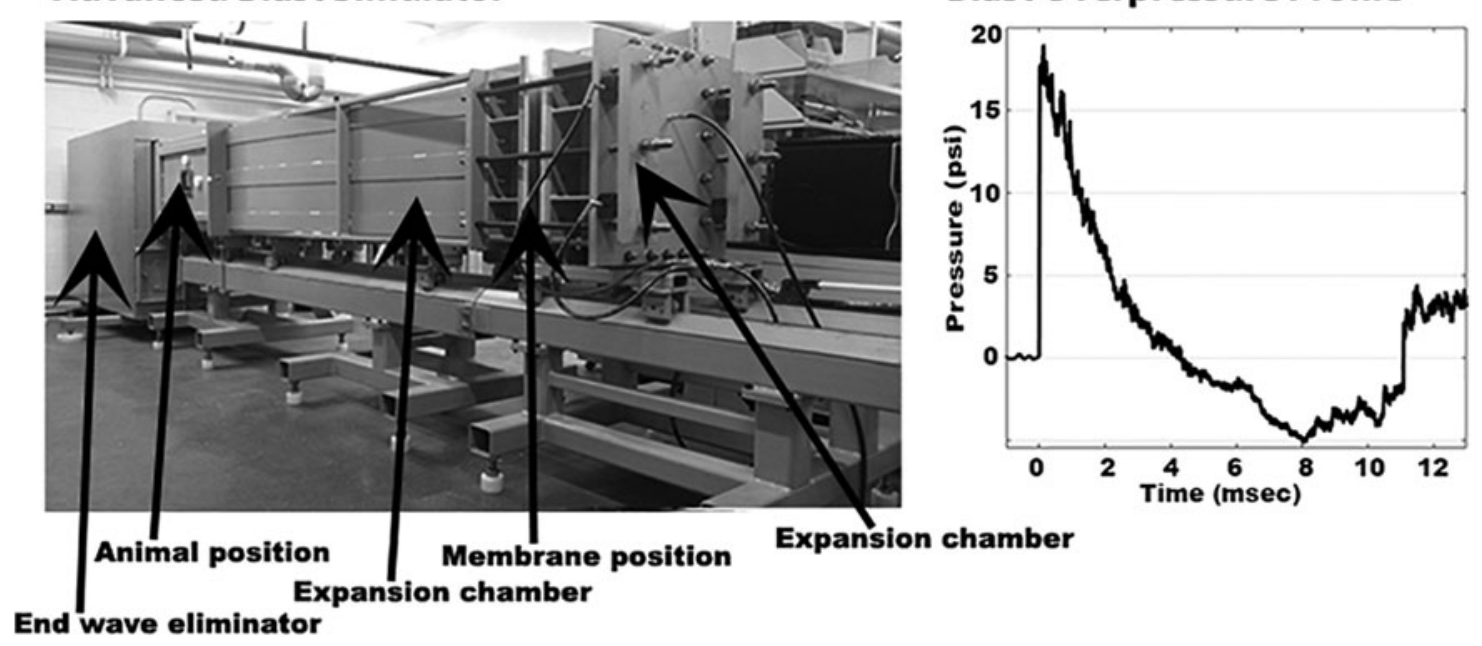

Blast Overpressure Profile

FIG. 1. The advanced blast simulator (ABS) used for the study (left) and the Friedlander blast overpressure wave generated in the ABS (right).

on Days 1, 6, and 14 and then Months 1,3, and 6 post-blast. The time taken from release to passing through the opening of the box was recorded. When the animal fell and failed to traverse the pole, the time and the location of the fall were recorded. Each run was scored using a protocol that incorporated balance (1 point for not falling; 0 points for a fall), velocity (distance on the pole covered/time) and distance completed (1 point for a complete run, 0.75 for a fall at the three-quarter mark, 0.5 for a fall in the middle and 0 points for a fall at the beginning). On each test day, each rat was given three trials. The best two (highest) scores were averaged as the score for that experimental subject on that day.

A total of 27 rats, with eight to nine rats in each group for different time-points, was used for the rotating pole test. At different time-points, some rats, including those from the sham controls did not want to perform the test and were excluded from the test for that time-point, which resulted in unequal number of rats in each group. This applied to other behavioral tests described below.

\section{Rotarod performance test}

The rotarod test was used to measure motor coordination after blast exposure. The rats undergoing the rotating pole test were not evaluated using rotarod performance as the latter test confounded performance on the former, and after training on the rotarod, rats would not traverse the pole, choosing instead to walk into the rotation based upon their experience with the rotarod. Consequently, separate sets of rats were evaluated using the rotarod test using a Rotamex Rotarod (Columbus Instruments, Columbus, $\mathrm{OH}$ ). Rats underwent 5 days of training before testing and a fixed speed of $20 \mathrm{rpm}$ was used for both the training and testing. Rats were given three trials per day during training and testing and each trial involved $3 \mathrm{~min}$. The latency to fall in each trial was monitored and the average value of the three trials (in sec) was used to assess the motor performance. Baseline testing was carried out on the day before blast exposure and the rats were tested again on Days 1, 6, and 14 and also at 1,3, and 6 months. Before retesting at 1, 3, and 6 months, the animals underwent 5 days of refresher training. A total of 45 rats, with eight to 15 rats in each group for different timepoints, was used for the rotarod performance test.

\section{Morris water maze test}

The Morris water maze (MWM) was used to evaluate spatial learning and long-term memory after blast exposure as described previously. ${ }^{16}$ The MWM test was performed in a $170-\mathrm{cm}$ diameter circular tub filled with water (maintained at $37-39^{\circ} \mathrm{C}$ ). The surface area of the water was divided into quadrants (NE, SE, NW, and $\mathrm{SW}$ ) of equal area by a video overlay recording system (PanLab, Barcelona, Spain). A clear transparent submerged square platform $\left(100 \mathrm{~cm}^{2}\right)$ was positioned beneath $(2 \mathrm{~cm})$ the surface of the water in the center of a quadrant. The MWM test was performed at 1 week and Months 1, 3, 6, 9, and 12 post-blast. There were 3 days of training/testing for the 1-week time-point, and for later times there were 2 days of training/testing. The first day of the 1-week training/test was used only for procedural acclimation and was not required for the other time-points.

Each day of training/testing consisted of four individual timed swims, each swim starting from a different cardinal compass location $(\mathrm{S}, \mathrm{N}, \mathrm{W}$, and $\mathrm{E})$ in the pool. On each test day, individual rats were removed from their home cages and placed into the water facing the wall of the pool at the cardinal compass location before being released. The order of releases were always $\mathrm{S}, \mathrm{N}, \mathrm{W}$, and $\mathrm{E}$. Each rat was allowed to swim for a maximum of $90 \mathrm{sec}$. If during this time, the rat located and mounted the platform, it was allowed to remain on it for $30 \mathrm{sec}$ before being dried and replaced in its home cage. Those not locating the platform were manually guided to the platform and allowed to remain on it for $30 \mathrm{sec}$ before starting another swim. Rats spent at least $5 \mathrm{~min}$ in their cages between trials. There was a total of 12 trials on the first day for each individual rat, which amounts to three rounds initiated from each of the four cardinal locations. Any rat showing signs of having difficulty swimming during the trial was removed before the $90 \mathrm{sec}$ has passed.

On the second day, individual rats were held against the wall of the pool, released at a cardinal location for a total of four trials. On the fifth trial, the platform was removed, and each rat was released from the NE wall and was allowed to swim for $30 \mathrm{sec}$. After $30 \mathrm{sec}$, the platform was placed in the SW quadrant and the rat was allowed to rest on the platform for $5 \mathrm{sec}$. On the third day, the platform was moved to the NE quadrant; each rat was held against the corresponding cardinal locations and released for a total of four trials to find the platform. The PanLab SMART video tracking system was used to record time taken to find the platform. A total of 40 rats, with 12 to 15 rats in each group for different time-points, was used for the MWM test.

\section{Novel object recognition test}

For measuring short-term memory, a novel object recognition (NOR) test was used as described previously. ${ }^{19}$ Rats used for 
MWM test were not used for NOR test to avoid complications resulting from rats undergoing too many handling and testing procedures. NOR test was performed on Day 8 and Months 1, 3, 6, and 12 post-blast. At each testing time-point, there were three phases. In the first phase, rats were acclimated to a custom-made testing chamber $(79 \times 79 \times 35 \mathrm{~cm})$ by allowing them to explore the empty chamber for $5 \mathrm{~min}$ for 3 consecutive days. After the first phase (acclimation period), the second phase involved presentation of two identical objects (glass bottles) in the chamber and the rats were allowed to explore them for $5 \mathrm{~min}$. Twenty minutes after the second phase, the third phase of testing occurred. In the third phase, one of the glass bottles was replaced with a glass bottle having different shape and the rats were allowed to explore the familiar and novel objects for $5 \mathrm{~min}$. The rats were monitored for time spent exploring the familiar and novel objects. Arena settings were set and behaviors were analyzed using SMART video tracking system and software (Harvard Apparatus, Holliston, MA). A discrimination index was calculated using the formula: (Time spent with novel object - Time spent with familiar object)/Total time spent with both objects. A total of 66 rats with 18 to 29 rats in each group for different time-points was used for the NOR test.

\section{Open field exploratory activity assessments}

Open field exploratory activity was measured using a locomotor activity apparatus (Omnitech Electronics, Columbus, $\mathrm{OH}$ ) as described earlier. ${ }^{30}$ Rats were allowed free exploration in the apparatus for $1 \mathrm{~h}$, and the activities such as total distance moved and time spent at the center and margins of the apparatus were measured using the Fusion Software (Omnitech Electronics, Columbus, $\mathrm{OH})$. The locomotor activities were monitored on Days 1, 6, and 14 and Months 1, 3, 6, 9, and 12 post-blast exposures. A total of 89 rats, with 10 to 31 rats in each group for different time-points, was used for the test.

\section{Statistical analysis}

Statistical analysis was carried out by two-way analysis of variance (ANOVA) followed by Tukey's post hoc test using honestly significant difference multiple comparisons (GraphPad Prism 6 software). Values were expressed as mean \pm standard error of the mean (SEM). When the data violated assumptions for ANOVA, non-normal distributed or with non-equal variances, KruskalWallis test was performed (GraphPad Prism 6 software). A $p$ value less than 0.05 was considered significant.

\section{Results}

Repeated blast exposure impaired vestibular motor functions in rats: Figure 2 shows the performance of sham controls and blast-exposed rats on the rotating pole. Performances of all the rats including sham controls deteriorated over time. At 6 months, all the rats, including sham controls, performed poorly and hence we terminated testing on the rotating pole past 6 months. Compared with sham controls, single blast-exposed animals showed limited statistically non-significant performance decrements on only Day 1 post-blast. Compared with sham controls and single blast-exposed animals, the repeated blast-exposed rats showed statistically significant (Kruskal-Wallis test) vestibular motor functional deficits on Days $6(p=0.013)$ and $14(p=0.025)$ and also at 1 month ( $p=0.015)$ post-blast exposures. Rats exposed to repeated blasts showed a functional recovery at 3 months postblast followed by a functional statistically non-significant decline again at 6 months compared with sham and single-blasted experimental subjects.

\section{Rotating pole test}

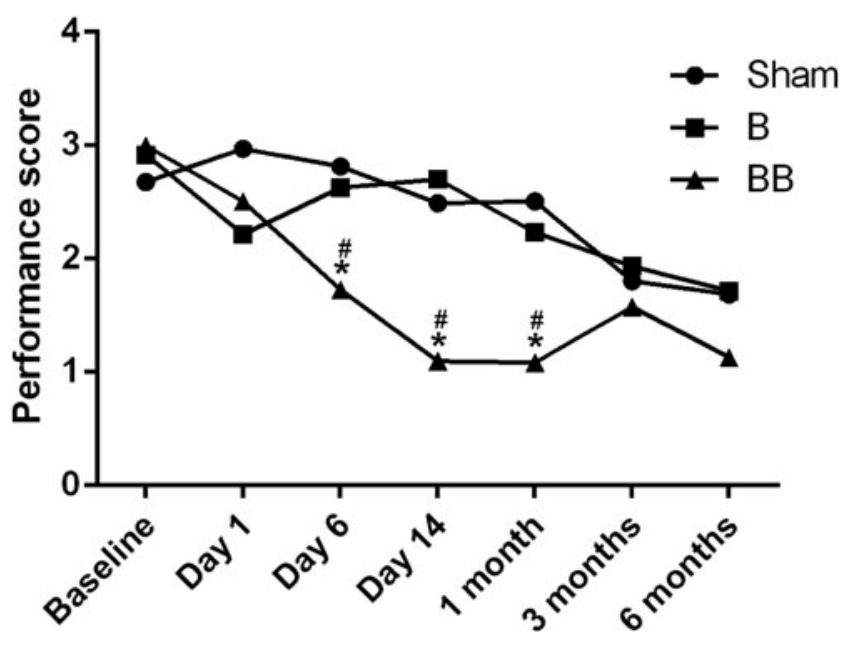

FIG. 2. The performance of rats on the rotating pole up to 6 months post-blast. Values are expressed as the average of the performance scores. Performance scores of sham animals were compared with those of both single and repeated blast-exposed animals ( ${ }^{*} p<0.05 ; n=8$ to 9$)$. Values of single blast-exposed animals were compared with those of repeated blast-exposed animals ( $\# p<0.05 ; n=8$ to 9$)$.

\section{Motor coordination is affected by repeated blast exposures}

As was seen with the rotating pole test, all rats including sham controls performed poorly on the rotarod at 6 months (Fig. 3), and consequently we terminated testing using the rotarod past 6 months post-blast. Rats exposed to repeated blast showed statistically significant [two-way ANOVA with multiple comparisons; $\mathrm{F}(2$, $230)=4.461 ; p=0.0126$ ] deficits on the rotarod on Day 6 and at

\section{Rotarod test}

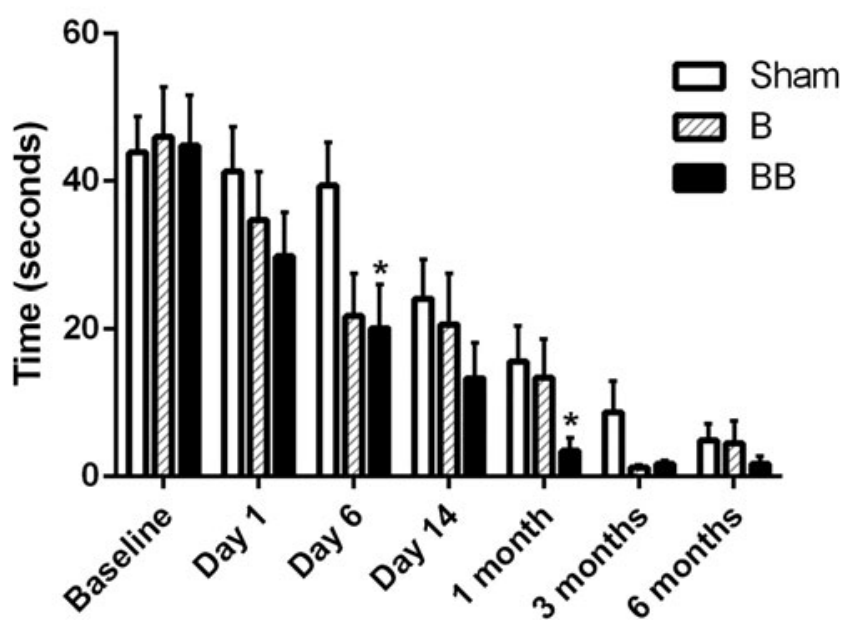

FIG. 3. The performance of rats on the rotarod up to 6 months post-blast. Values are expressed as mean \pm standard error of the mean. The latency (in sec) to fall off the rotarod of sham animals was compared with that of both single and repeated blast-exposed animals $\left({ }^{*} p<0.05 ; n=8\right.$ to 15$)$. No statistically significant changes were observed between single and repeated blast-exposed animals at any of the time-points evaluated. 
1 month post-blast exposures compared with sham controls. In contrast, rats exposed to a single blast did not show statistically significant deficits compared with sham controls at any of the timepoints evaluated, although their performances were generally diminished at Day 6 and at 3 months.

Spatial learning and memory deficits were observed in rats exposed to single and repeated blasts: MWM test for spatial learning and memory revealed a biphasic change in functions with an acute deficit followed by a recovery at sub-acute time-points and a significant deficit at 9 months after repeated blast exposures [Fig. 4; two-way ANOVA with multiple comparisons; $\mathrm{F}(2,167)=6.766$; $p=0.0015]$. The acute deficit in spatial learning and memory, determined by the time required to find the platform, was observed in the rats exposed to repeated blasts compared with both sham controls and single blast-exposed rats $(p<0.01)$. Compared with sham controls, memory deficits were observed in both single $(p<0.001)$ and repeated $(p<0.05)$ blast-exposed rats at 9 months post-blast. No significant functional deficits in spatial learning and long-term memory were observed at $1,3,6$, or 12 months post-blast.

\section{Single and repeated blast exposures affected short-term memory}

The novel object recognition test revealed loss of short-term memory in rats after single and repeated blast exposures (Fig. 5). In sham controls, the discrimination index did not reveal significant changes across the different time-points evaluated; consequently, the values of all the time-points were pooled together. The discrimination index values of single and repeated blast-exposed rats were significantly lower than sham controls at 6 and 12 months suggesting a loss of short-term memory at later time-points after blast exposures [two-way ANOVA with multiple comparisons; $\mathrm{F}(2,248)=3.652 ; p=0.0274]$.

Single and repeated blast-exposed animals developed anxiety and depressive-like behaviors at acute and chronic time-points

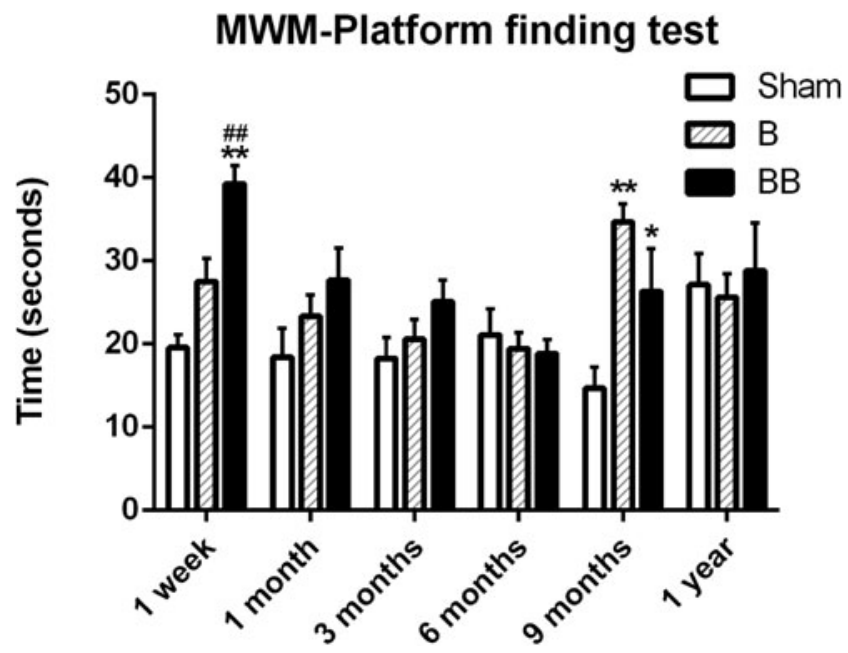

FIG. 4. The performance of rats in the platform finding test of MWM from 1 week to 1 year post-blast. The values are the average of the four trials carried out on the test day. Values are expressed as mean \pm standard error of the mean. The time taken by the sham animals to find the platform was compared with that of both single and repeated blast-exposed animals $\left({ }^{*} p<0.05\right.$, $* * p<0.001 ; n=12$ to 15$)$. The time taken by the single blastexposed animals to find the platform was compared with that of repeated blast-exposed animals (\#\#p $<0.001 ; n=12$ to 15 ).

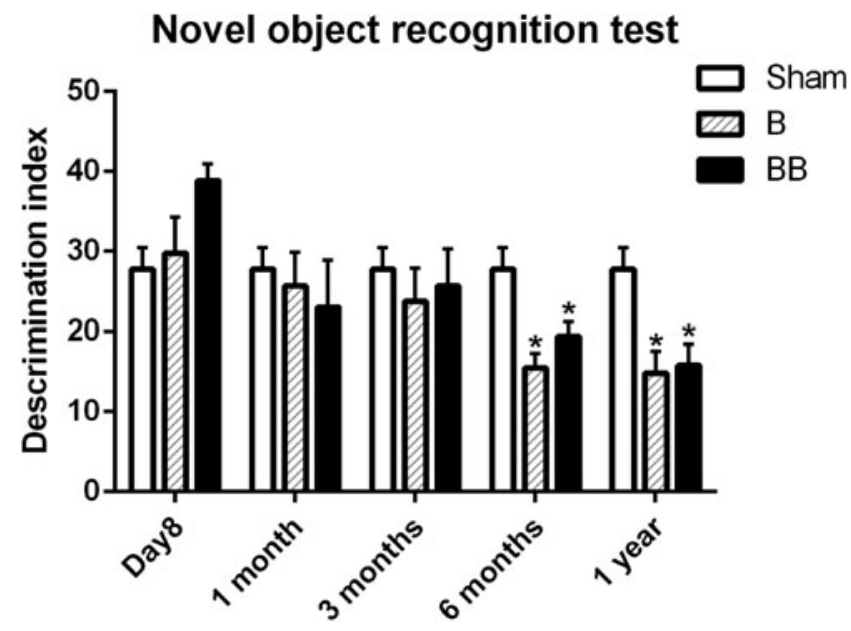

FIG. 5. The performance of rats in the NOR test for short-term memory from 1 week to 1 year post-blast. The discrimination index values are expressed as mean \pm SEM. The discrimination index values of sham animals were compared with those of both single and repeated blast-exposed animals ( ${ }^{*} p<0.05, n=18$ to 29$)$. No statistically significant changes were observed between single and repeated blast-exposed animals at any of the time-points evaluated.

post-blasts: Open field exploratory activities also showed biphasic changes in functional outcomes after blast exposures (Fig. 6A-C). All three functional parameters evaluated using open field activity showed acute changes followed by a recovery and subsequent significant changes observed again after 6,9 , and 12 months post-blast exposures. The total distance moved, a measure of the overall locomotion activity, showed statistically significant changes at different time-points [two-way ANOVA with multiple comparisons; $\mathrm{F}(2,529)=23.64 ; p=0.0001]$. The total distance moved was significantly decreased on day 1 after repeated blast exposures compared with both sham controls and single blast $(p<0.01)$ exposed animals. Compared with sham controls, the repeated blast-exposed rats showed significant decreases in the total distance travelled again at $3(p<0.05), 6(p<0.05), 9(p<0.01)$ and $12(p<0.01)$ months, whereas the single blast-exposed rats showed significant decreases only at $6(p<0.05)$ and $9(p<0.05)$ months. Compared with single blast-exposed rats, those rats exposed to repeated blasts showed significant decreases in total distance travelled on Days $1(p<0.01)$ and $14(p<0.05)$, as well as at $3(p<0.01), 9(p<0.05)$ and $12(p<0.05)$ months post-blast. When compared with sham controls, rats exposed to single and repeated blast did not show significant changes in total distance travelled on Days 6 and 14 as well as at 1 month.

Center time and margin time also showed biphasic changes after single and repeated blast exposures [two-way ANOVA with multiple comparisons; $\mathrm{F}(2,529)=22.52 ; p=0.0001]$. Compared with both sham controls and single blast-exposed animals, the rats exposed to repeated blasts spent less time exploring the center of the arena on Days $1(p<0.05), 6(p<0.01)$ and $14(p<0.01)$, but did not show any significant changes from the other two treatment groups at 1 and 3 months. Repeated blast-exposed animals again showed significantly less center time at 6, 9, and 12 months compared with sham controls $(p<0.01)$. Single blast-exposed animals also spent less time exploring the center of the arena on Days 1 and 14 and again at 6,9 , and 12 months post-blast compared with sham controls $(p<0.05)$. Margin time results reveal that the blastexposed animals spent more time exploring the perimeter of the arena compared with sham controls [two-way ANOVA with 

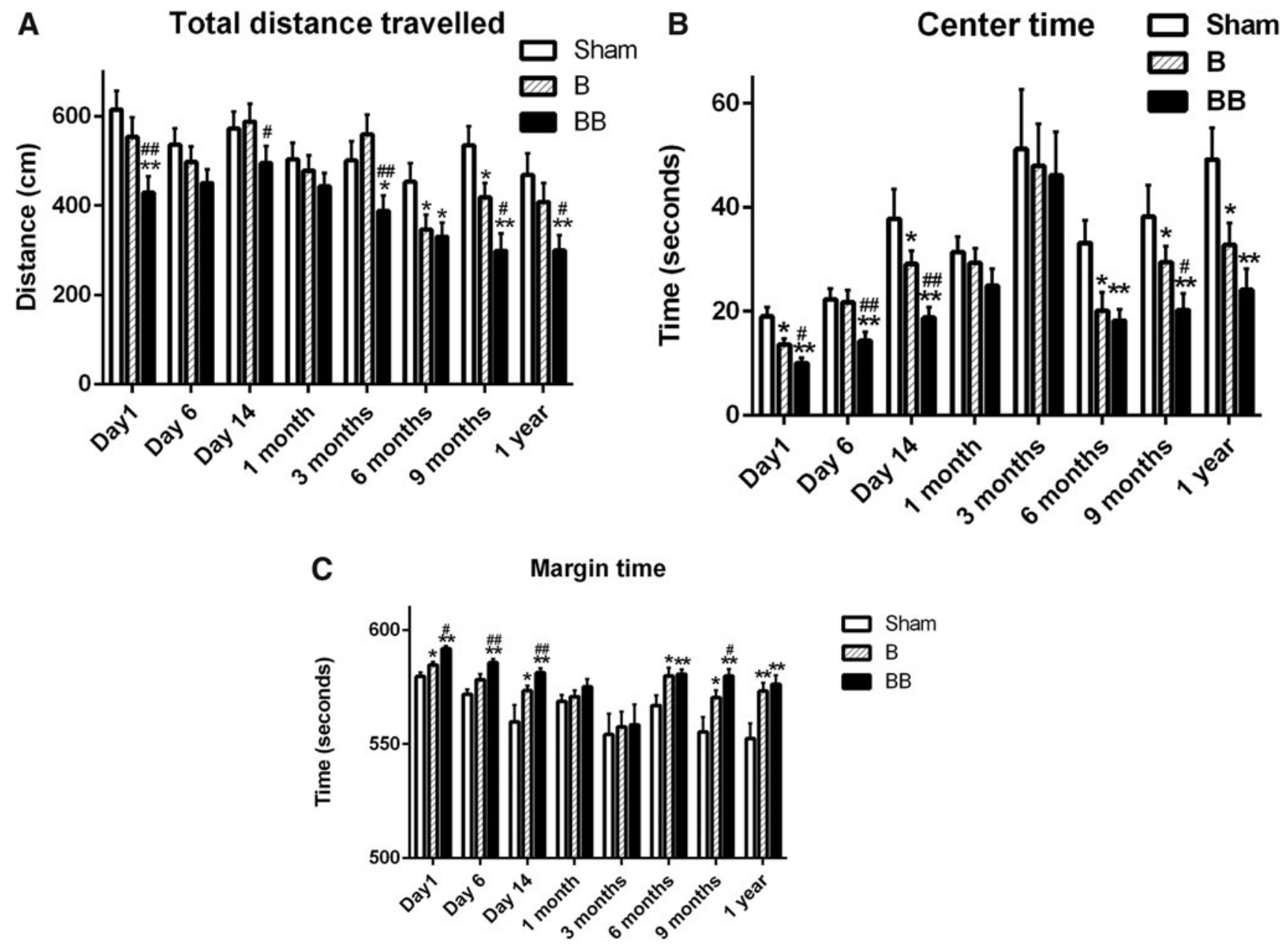

FIG. 6. Open filed exploratory activities of rats from Day 1 up to 1 year post-blast. (A) The total distance the animals moved in the open field arena in $1 \mathrm{~h}$. (B) The total time the rats spent near the center of the arena in $1 \mathrm{~h}$. (C) The total time the animals spent at the perimeter of the arena in $1 \mathrm{~h}$. Values are expressed as mean \pm standard error of the mean. The values of sham rats were compared with that of both single and repeated blast-exposed rats $\left({ }^{*} p<0.05,{ }^{*} p<0.01 ; n=10-31\right)$. The values of single blast-exposed rats were compared with those of repeated blast-exposed rats $(\# p<0.05, \# \#<0.01 ; n=10-31)$.

multiple comparisons; $\mathrm{F}(2,529)=29.87 ; p=0.0001]$. Compared with both sham controls and single blast-exposed animals, the repeated blast-exposed rats spent significantly more time exploring the margins of the arena on Days $1(p<0.05), 6(p<0.01)$, and 14 $(p<0.01)$, but did not show similar differences at 1 and 3 months. Repeated blast-exposed rats again showed significantly more margin time at 6, 9, and 12 months compared with sham controls $(p<0.01)$. Compared with sham controls, single blast-exposed animals spent more time exploring the margins of the arena on Days 1 and $14(p<0.05)$ and again at $6(p<0.05), 9(p<0.05)$, and 12 $(p<0.01)$ months post-blast. Finally, at 9 months post-blast, the repeated blast-exposed animals showed significantly higher margin time compared with single blast-exposed animals $(p<0.05)$.

\section{Discussion}

Exposure to single and closely coupled blasts resulted in acute neurobehavioral functional deficits, most of which diminished after several weeks but then deteriorated again after 6 months post-blast. This biphasic pattern of neurobehavioral changes after single or repeated blast exposures has not been reported previously. An earlier longitudinal study employing a single blast exposure of 17 psi showed sustained neurobehavioral deficits at both 1 and 3 months, which were the only times of assessments. ${ }^{17}$ In that study, the novel object recognition test (for short-term memory) and light/dark box test (for anxiety and active avoidance) were performed. In the present study, we have expanded evaluations to test the effects of both single and repeated blast exposures on neuromotor functions, short-/long-term memory, anxiety and depressivelike behaviors at acute, sub-acute, and chronic time-points up to 1 year.

For repeated exposures, we applied two tightly coupled blasts within a 2-min interval similar to what we have reported previously. ${ }^{25}$ Exposure to repeated shock waves in this manner is not uncommon either operationally or in military training and generates a more robust insult to the central nervous system that is relatively free of complicating polytrauma than is possible with a single exposure to a higher amplitude waveform. In addition to improvised explosive device detonations, high overpressure weapon systems, such as artillery, can produce closely coupled consecutive overpressure-based insults. One practical advantage of using the tightly coupled blast is that it eliminates the need for an additional isoflurane anesthetization, which is known to be neuroprotective against TBI. ${ }^{31,32}$ 
Using the rotating pole and rotarod tests to evaluate balance and coordination, we observed that all the experimental subjects, including the sham controls, performed poorly at 6 months postblast(s). Thus, testing at 9 and 12 months was discontinued to reduce unnecessary stress to the animals. The deterioration in performance at 6 months could not be simply attributed to an increase in body weight since diet restrictions across all treatment groups maintained body weights between 450 and $500 \mathrm{~g}$ from 1 month to 12 months. It is likely that habituation played a significant role in the performance of rats including the sham controls on the rotating pole and rotarod at later time-points. The performance of the sham controls, which showed a steady decrease after the first week of testing, points to a possible confounding testing habituation. Over the abbreviated testing schedule, rats exposed to a single blast did not show functional deficits relative to shams with either test (Fig. 2 and Fig. 3). In contrast, rats exposed to tightly coupled repeated blasts had significant neuro-motor dysfunction, with deficiencies in balance and co-ordination at Days 6 and 14 as well as at 1 month post-blast exposures (Fig. 2 and Fig. 3).

Although the rats exposed to repeated blasts showed a recovery at 3 months, there was a statistically non-significant trend towards deterioration again at 6 months on the rotating pole (Fig. 2). Overall, we generally found the rotating pole test to be more sensitive than the rotarod test to distinguish neuro-motor dysfunction after blast exposure, and results from prior studies of blast-induced TBI employing a rotarod are somewhat mixed. Evaluations made at acute and subacute time-points showed no neuro-motor functional deficits in the rotarod test after single blast exposures outside of a cylindrical shock tube ${ }^{33}$ with longitudinal animal orientation to the oncoming shock waves similar to that used in the present study. In contrast, we observed that both single and repeated blast exposures of mice transversely positioned in a cylindrical shock tube caused acute and subacute neuro-motor functional deficits in the rotarod test. ${ }^{14}$

Apart from the differences in species and orientations, different outcomes in these experiments are likely greatly influenced by the vastly different flow conditions created in these different types of blast simulators, with artefactual loading conditions likely contributing substantially to injuries and impairments produced in constant diameter shock tubes. In contrast, through incorporation of a divergent transition section, the ABS used in the current study produces a fidelic Friedlander waveform and an appreciably diminished dynamic pressure (blast wind), which can greatly contribute to loading conditions and the injuries produced. ${ }^{26}$ No prior studies have evaluated neuro-motor functional deficits using these functional tests (rotarod and rotating pole tests) after exposure to single or repeated blasts using the ABS design for blast simulation in the laboratory. Long-term problems in neuro-motor functions after primary blast exposure is consistent with the chronic neuro-motor dysfunctions reported in active duty and retired service members. ${ }^{34-36}$

The MWM test used to evaluate long-term memory functions showed significant changes at 1 week and 9 months post-blast (Fig. 4). Compared with sham controls, repeated blast-exposed animals showed spatial learning and memory deficiencies at 1 week and 9 months, but single blast-exposed animals showed significant deficits only at 9 months. A recent study employing evaluations up to 2 weeks after a single blast exposure in an ABS also did not detect significant functional deficits in the MWM test. ${ }^{37}$ In contrast, previous studies carried out using constant diameter cylindrical shock tubes reported significant spatial learning and memory deficits in rats at acute time-points after single blast exposure. ${ }^{16,38}$ As described above, the disparities in the results obtained in this study using an ABS and previous studies using cylindrical shock tubes could be due to the blast loading artifacts encountered in the constant diameter shock tubes and also to differences in the positioning orientations of the animals against the oncoming shock waves.

Our data showing long-term memory problems in rats at acute and chronic time-points after blast exposure are consistent with the clinical data showing significant memory deficits in soldiers exposed to blast waves. ${ }^{39-43}$ In general, after closely coupled repeated blast exposures, sensorimotor functions in rats (e.g., rotating pole) normalized by 3 months whereas behavioral abnormalities reflecting memory, anxiety, and/or depression were more robust and persistent, lasting through 9-12 months. These findings loosely parallel observations made among warfighters injured by blast in the wars in Iraq and Afghanistan, where, despite self-reported memory complaints, individuals generally perform well on objective measures of memory, yet display significant persistent behavioral/PTSD-related symptoms. ${ }^{44-46}$ "Blast-induced PTSD-related traits" have recently been described by others in rats and were similarly shown to persist through 8 months following blast exposure. ${ }^{23}$

The NOR test was widely used for evaluating short- and longterm memory functions in experimental animals after blast exposure. ${ }^{17,19,47,48}$ The data obtained from the NOR test in the present study showed statistically significant differences only at 6 and 12 months post-blast (Fig. 5). In contrast, a previous study in which rats were evaluated up to 3 months after a single 17-psi blast exposure in an ABS reports significant short-term memory deficits in the NOR test at both 1 and 3 months post-blast. ${ }^{17}$ The results obtained from the MWM and NOR tests suggest that both short and long-term memory defects occur after primary blast exposure. This is noteworthy since acute and long-term defects in memory are major concerns in active duty and retired service members, and several clinical studies have reported memory problems in service members after blast exposure which deteriorated with time. ${ }^{39-43}$ Recent clinical evaluations using magnetic resonance spectroscopic imaging revealed a significant decrease in the ratios of $\mathrm{N}$ acetyl aspartate to both choline and creatine in the anterior portions of hippocampus in association with significant visual memory deficits. ${ }^{43}$ Potentially, available therapeutic interventions at acute stages after blast exposure may prevent or decrease severe cognitive dysfunctions developing at later stages of life.

Anxiety, depression, and suicidal activities are major concerns among active duty and retired service members. Blast-exposed service members reported to have long-term anxiety and depression symptoms. ${ }^{49,50}$ Many neuropsychiatric abnormalities associated with blast exposure overlap with those of PTSD. ${ }^{49,51}$ In the current study, we have shown that blast exposure resulted in biphasic changes in anxiety and depressive-like behaviors in rats and the severity increased with repeated blast exposures (Fig. 6A-C). These rats travelled less distance in the open field apparatus, spent more time at the margins and travelled less time through the center of the arena, all of which are thought to be indicators of anxiety and depressive-like traits. These changes were most pronounced at later time-points (past 6 months), which is consistent with the symptoms of longterm psychiatric problems observed in blast-exposed victims. ${ }^{49,50}$

Prior pre-clinical studies with constant diameter shock tubes also reported anxiety and depressive-like behaviors at acute and subacute time-points. ${ }^{12,52}$ Mice exposed to a single blast with overpressures greater than those in the present study also spent more time at the margins of the open field arena at acute and sub-acute time-points-interpreted as a freezing response and lack of interest towards the environment, which is a behavior comparable to that described with post-traumatic depression in humans. ${ }^{12}$ The changes observed in those mice were blast overpressure dose dependent. In 
contrast, repeated, but not single, blast exposures resulted in anxiety-related behavior at 1 day, but not at 16 days in rats exposed to mild blast overpressure waves in a cylindrical shock tube. ${ }^{52}$

Repeated low-level blast exposure have also have elicited acute and chronic anxiety- and PTSD-like symptoms in rats. ${ }^{22,23}$ In those particular studies, the rats were exposed to one 10.8 psi blast each day for 3 consecutive days using a cylindrical shock tube and evaluated employing a number of neurobehavioral tests for anxiety and PTSDlike traits up to 35 weeks post-blasts. Replication of these studies with the blast insult produced by an ABS would be of value to consolidate findings across laboratories and methodologies. The present study reveals that a single moderate-level blast exposure using the ABS elicited behaviors thought to reflect anxiety and depression at acute time-points, followed by a transient recovery during the sub-acute time-points, and a subsequent deterioration again after 6 months through 1 year. The magnitude of these changes was further increased following closely coupled repeated blast exposures.

Dietary restriction to limit weight gain over the course of the study may have introduced a confounding influence/variable in light of several reports of salutary effects of dietary restriction on recovery and outcomes in other models of traumatic brain injury. ${ }^{53,54}$ Although diets were identically controlled in both shaminjured and blast-injured rats in the current study, it is conceivable that the magnitude of impairments recorded over the course of the study may have been diminished by the dietary restriction imposed for weight control. Nevertheless, changes were recorded largely unencumbered by confounding obesity.

In conclusion, the ABS generates blast overpressure waves closely resembling those seen with free field explosions, which cause significant neurobehavioral deficits at acute and chronic time-points, particularly with closely coupled exposures. Several neurobehavioral functional defects at chronic stages are more pronounced than are observed at acute time-points, suggesting the importance of evaluating animals longitudinally for several months after blast exposure. These apparently biphasic changes also point to the importance of continued care after initial symptomatic treatments period post-blast exposure. Studies are currently underway to identify the pathological mechanisms underlying biphasic functional changes, which is essential for rationally developing countermeasures to the later stage deficits resulting from blast exposure.

\section{Acknowledgments}

The technical help received from Meghan Benton, Chloe Christoforou and Wafae Driwech at the initial stages of this study is gratefully acknowledged. The authors thank Dr. Yuanzhang Li for statistical analysis and data interpretation.

The preliminary results of this study were submitted as an abstract to the 35th National Neurotrauma Symposium held at Snowbird, UT, and the abstract was published in Journal of Neurotrauma, 34, A-1-A-164 (July 1, 2017).

The contents, opinions, and assertions contained herein are private views of the authors and are not to be construed as official or reflecting the views of the Department of the Army or the Department of Defense.

\section{Funding Information}

This study was funded by the Military Operational Medicine Research Program at United States Army Medical Research and Materiel Command.

\section{Author Disclosure Statement}

No competing financial interests exist.

\section{References}

1. Reid, M.W., Miller, K.J., Lange, R.T., Cooper, D.B., Tate, D.F., Bailie, J., Brickell, T.A., French, L.M., Asmussen, S., and Kennedy, J.E. (2014). A multisite study of the relationships between blast exposures and symptom reporting in a post-deployment active duty military population with mild traumatic brain injury. J. Neurotrauma 31, 1899-1906.

2. DePalma, R.G., Burris, D.G., Champion, H.R., and Hodgson, M.J. (2005). Blast injuries. N. Engl. J. Med. 352, 1335-1342.

3. Warden, D. (2006). Military TBI during the Iraq and Afghanistan wars. J. Head Trauma Rehabil. 21, 398-402.

4. Kontos, A.P., Elbin, R.J., Kotwal, R.S., Lutz, R.H., Kane, S., Benson, P.J., Forsten, R.D., and Collins, M.W. (2015). The effects of combatrelated mild traumatic brain injury (mTBI): does blast mTBI history matter? J. Trauma Acute Care Surg. 79, S146-S151.

5. Fear, N.T., Jones, E., Groom, M., Greenberg, N., Hull, L., Hodgetts, T.J., and Wessely, S. (2009). Symptoms of post-concussional syndrome are non-specifically related to mild traumatic brain injury in UK Armed Forces personnel on return from deployment in Iraq: an analysis of self-reported data. Psychol. Med. 39, 1379-1387.

6. Levin, H.S., Wilde, E., Troyanskaya, M., Petersen, N.J., Scheibel, R., Newsome, M., Radaideh, M., Wu, T., Yallampalli, R., Chu, Z., and Li, X. (2010). Diffusion tensor imaging of mild to moderate blast-related traumatic brain injury and its sequelae. J. Neurotrauma 27, 683-694.

7. Vanderploeg, R.D., Curtiss, G., Luis, C.A., and Salazar, A.M. (2007). Long-term morbidities following self-reported mild traumatic brain injury. J. Clin. Exp. Neuropsychol. 29, 585-598.

8. Hoge, C.W., McGurk, D., Thomas, J.L., Cox, A.L., Engel, C.C., and Castro, C.A. (2008). Mild traumatic brain injury in U.S. Soldiers returning from Iraq. N. Engl. J. Med. 358, 453-463.

9. Kontos, A.P., Kotwal, R.S., Elbin, R.J., Lutz, R.H., Forsten, R.D., Benson, P.J., and Guskiewicz, K.M. (2013). Residual effects of combat-related mild traumatic brain injury. J. Neurotrauma 30, 680686.

10. Taber, K.H., Hurley, R.A., Haswell, C.C., Rowland, J.A., Hurt, S.D., Lamar, C.D., and Morey, R.A. (2015). White matter compromise in veterans exposed to primary blast forces. J. Head Trauma Rehabil. 30, E15-E25.

11. Trotter, B.B., Robinson, M.E., Milberg, W.P., McGlinchey, R.E., and Salat, D.H. (2015). Military blast exposure, ageing and white matter integrity. Brain 138, 2278-2292.

12. Cernak, I., Merkle, A.C., Koliatsos, V.E., Bilik, J.M., Luong, Q.T., Mahota, T.M., Xu, L., Slack, N., Windle, D., and Ahmed, F.A. (2011). The pathobiology of blast injuries and blast-induced neurotrauma as identified using a new experimental model of injury in mice. Neurobiol. Dis. 41, 538-551.

13. Koliatsos, V.E., Cernak, I., Xu, L., Song, Y., Savonenko, A., Crain, B.J., Eberhart, C.G., Frangakis, C.E., Melnikova, T., Kim, H., and Lee, D. (2011). A mouse model of blast injury to brain: initial pathological, neuropathological, and behavioral characterization. J. Neuropathol. Exp. Neurol. 70, 399-416.

14. Wang, Y., Wei, Y., Oguntayo, S., Wilkins, W., Arun, P., Valiyaveettil, M., Song, J., Long, J., and Nambiar, M.P. (2011). Tightly coupled repetitive blast-ind,uced traumatic brain injury: development and characterization in mice. J. Neurotrauma 28, 2171-2183.

15. Muelbl, M.J., Slaker, M.L., Shah, A.S., Nawarawong, N.N., Gerndt, C.H., Budde, M.D., Stemper, B.D., and Olsen, C.M. (2018). Effects of mild blast traumatic brain injury on cognitive- and addiction-related behaviors. Sci. Rep. 8, 9941.

16. Long, J.B., Bentley, T.L., Wessner, K.A., Cerone, C., Sweeney, S., and Bauman, R.A. (2009). Blast overpressure in rats: recreating a battlefield injury in the laboratory. J. Neurotrauma 26, 827-840.

17. Sajja, V.S., Hubbard, W.B., Hall, C.S., Ghoddoussi, F., Galloway, M.P., and VandeVord, P.J. (2015). Enduring deficits in memory and neuronal pathology after blast-induced traumatic brain injury. Sci. Rep. 5, 15075.

18. Kovesdi, E., Gyorgy, A.B., Kwon, S.K., Wingo, D.L., Kamnaksh, A., Long, J.B., Kasper, C.E., and Agoston, D.V. (2011). The effect of enriched environment on the outcome of traumatic brain injury; a behavioral, proteomics, and histological study. Front. Neurosci. 5, 42. 
19. Cho, H.J., Sajja, V.S., Vandevord, P.J., and Lee, Y.W. (2013). Blast induces oxidative stress, inflammation, neuronal loss and subsequent short-term memory impairment in rats. Neuroscience 253, 9-20.

20. Xie, K., Kuang, H., and Tsien, J.Z. (2013). Mild blast events alter anxiety, memory, and neural activity patterns in the anterior cingulate cortex. PLoS One 8, e64907.

21. Sajja, V.S., Hubbard, W.B., and VandeVord, P.J. (2015). Subacute oxidative stress and glial reactivity in the amygdala are associated with increased anxiety following blast neurotrauma. Shock 44 Suppl 1, 71-78.

22. Perez-Garcia, G., Gama Sosa, M.A., De Gasperi, R., Lashof-Sullivan, M., Maudlin-Jeronimo, E., Stone, J.R., Haghighi, F., Ahlers, S.T., and Elder, G.A. (2018). Chronic post-traumatic stress disorder-related traits in a rat model of low-level blast exposure. Behav. Brain Res. 340, 117-125.

23. Perez-Garcia, G., Gama Sosa, M.A., De Gasperi, R., Tschiffely, A.E., McCarron, R.M., Hof, P.R., Gandy, S., Ahlers, S.T., and Elder, G.A. (2019). Blast-induced "PTSD": evidence from an animal model. Neuropharmacology 145, 220-229.

24. Perez-Garcia, G., De Gasperi, R., Gama Sosa, M.A., Perez, G.M., Otero-Pagan, A., Tschiffely, A., McCarron, R.M., Ahlers, S.T., Elder, G.A., and Gandy, S. (2018). PTSD-related behavioral traits in a rat model of blast-induced mTBI are reversed by the $m$ GluR2/3 receptor antagonist BCI-838. eNeuro 5.

25. Arun, P., Rittase, W.B., Wilder, D.M., Wang, Y., Gist, I.D., and Long, J.B. (2018). Defective methionine metabolism in the brain after repeated blast exposures might contribute to increased oxidative stress. Neurochem. Int. 112, 234-238.

26. Needham, C.E., Ritzel, D., Rule, G.T., Wiri, S., and Young, L. (2015). Blast testing issues and TBI: experimental models that lead to wrong conclusions. Front. Neurol. 6, 72 .

27. Sawyer, T., Wang, Y., Ritzel, D.V., Josey, T., Villanueva, M., Shei, Y., Nelson, P., Hennes, G., Weiss, T., Vair, C., Fan, C., and Barnes, J. (2015). High fidelity simulation of primary blast: direct effects on the head. J. Neurotrauma 33, 1181-1193.

28. Vu, P.A., Tucker, L.B., Liu, J., McNamara, E.H., Tran, T., Fu, A.H., Kim, Y., and McCabe, J.T. (2018). Transient disruption of mouse home cage activities and assessment of orexin immunoreactivity following concussive- or blast-induced brain injury. Brain Res. 1700, 138-151.

29. Mattiasson, G.J., Philips, M.F., Tomasevic, G., Johansson, B.B., Wieloch, T., and McIntosh, T.K. (2000). The rotating pole test: evaluation of its effectiveness in assessing functional motor deficits following experimental head injury in the rat. J. Neurosci. Methods 95, 75-82.

30. Arun, P., Ariyannur, P.S., Moffett, J.R., Xing, G., Hamilton, K., Grunberg, N.E., Ives, J.A., and Namboodiri, A.M. (2010). Metabolic acetate therapy for the treatment of traumatic brain injury. J. Neurotrauma 27, 293-298.

31. Statler, K.D., Alexander, H., Vagni, V., Holubkov, R., Dixon, C.E., Clark, R.S., Jenkins, L., and Kochanek, P.M. (2006). Isoflurane exerts neuroprotective actions at or near the time of severe traumatic brain injury. Brain Res. 1076, 216-224.

32. Statler, K.D., Kochanek, P.M., Dixon, C.E., Alexander, H.L., Warner, D.S., Clark, R.S., Wisniewski, S.R., Graham, S.H., Jenkins, L.W., Marion, D.W., and Safar, P.J. (2000). Isoflurane improves long-term neurologic outcome versus fentanyl after traumatic brain injury in rats. J. Neurotrauma 17, 1179-1189.

33. Beamer, M., Tummala, S.R., Gullotti, D., Kopil, C., Gorka, S., Ted, A., Bass, C.R., Morrison, B., 3rd, Cohen, A.S., and Meaney, D.F. (2016). Primary blast injury causes cognitive impairments and hippocampal circuit alterations. Exp. Neurol. 283, 16-28.

34. Gardner, R.C., Peltz, C.B., Kenney, K., Covinsky, K.E., Diaz-Arrastia, R., and Yaffe, K. (2017). Remote traumatic brain injury is associated with motor dysfunction in older military veterans. J. Gerontol. A Biol. Sci. Med. Sci. 72, 1233-1238.

35. Hoffer, M.E., Balaban, C., Gottshall, K., Balough, B.J., Maddox, M.R., and Penta, J.R. (2010). Blast exposure: vestibular consequences and associated characteristics. Otol. Neurotol. 31, 232-236.

36. Akin, F.W. and Murnane, O.D. (2011). Head injury and blast exposure: vestibular consequences. Otolaryngol. Clin. North Am. 44, 323-334, viii.

37. Rodriguez, U.A., Zeng, Y., Deyo, D., Parsley, M.A., Hawkins, B.E., Prough, D.S., and DeWitt, D.S. (2018). Effects of mild blast traumatic brain injury on cerebral vascular, histopathological, and behavioral outcomes in rats. J. Neurotrauma 35, 375-392.

38. Budde, M.D., Shah, A., McCrea, M., Cullinan, W.E., Pintar, F.A., and Stemper, B.D. (2013). Primary blast traumatic brain injury in the rat: relating diffusion tensor imaging and behavior. Front. Neurol. 4, 154.
39. Hoffer, M.E., Balaban, C., Slade, M.D., Tsao, J.W., and Hoffer, B. (2013). Amelioration of acute sequelae of blast induced mild traumatic brain injury by $\mathrm{N}$-acetyl cysteine: a double-blind, placebo controlled study. PLoS One 8, e54163.

40. Graner, J., Oakes, T.R., French, L.M., and Riedy, G. (2013). Functional MRI in the investigation of blast-related traumatic brain injury. Front. Neurol. 4, 16.

41. Bogdanova, Y. and Verfaellie, M. (2012). Cognitive sequelae of blastinduced traumatic brain injury: recovery and rehabilitation. Neuropsychol. Rev. 22, 4-20.

42. Mac Donald, C.L., Barber, J., Andre, J., Evans, N., Panks, C., Sun, S. Zalewski, K., Elizabeth Sanders, R., and Temkin, N. (2017). 5-Year imaging sequelae of concussive blast injury and relation to early clinical outcome. Neuroimage Clin. 14, 371-378.

43. de Lanerolle, N.C., Hamid, H., Kulas, J., Pan, J.W., Czlapinski, R., Rinaldi, A., Ling, G., Bandak, F.A., and Hetherington, H.P. (2014) Concussive brain injury from explosive blast. Ann. Clin. Transl. Neurol. 1, 692-702.

44. Pagulayan, K.F., Petrie, E.C., Cook, D.G., Hendrickson, R.C., Rau, H., Reilly, M., Mayer, C., Meabon, J.S., Raskind, M.A., Peskind, E.R. and Kleinhans, N. (2018). Effect of blast-related mTBI on the working memory system: a resting state fMRI study. Brain Imaging Behav.

45. Miller, D.R., Hayes, J.P., Lafleche, G., Salat, D.H., and Verfaellie, M. (2017). White matter abnormalities are associated with overall cognitive status in blast-related mTBI. Brain Imaging Behav. 11, 1129-1138.

46. Troyanskaya, M., Pastorek, N.J., Scheibel, R.S., Petersen, N.J., McCulloch, K., Wilde, E.A., Henson, H.K., and Levin, H.S. (2015). Combat exposure, PTSD symptoms, and cognition following blastrelated traumatic brain injury in OEF/OIF/OND service members and veterans. Mil. Med. 180, 285-289.

47. Rubovitch, V., Zilberstein, Y., Chapman, J., Schreiber, S., and Pick, C.G. (2017). Restoring GM1 ganglioside expression ameliorates axonal outgrowth inhibition and cognitive impairments induced by blast traumatic brain injury. Sci. Rep. 7, 41269.

48. Rachmany, L., Tweedie, D., Rubovitch, V., Li, Y., Holloway, H.W., Kim, D.S., Ratliff, W.A., Saykally, J.N., Citron, B.A., Hoffer, B.J., Greig, N.H., and Pick, C.G. (2017). Exendin-4 attenuates blast traumatic brain injury induced cognitive impairments, losses of synaptophysin and in vitro TBI-induced hippocampal cellular degeneration. Sci. Rep. 7, 3735.

49. Rosenfeld, J.V. and Ford, N.L. (2010). Bomb blast, mild traumatic brain injury and psychiatric morbidity: a review. Injury 41, 437-443.

50. Walker, W.C., Franke, L.M., McDonald, S.D., Sima, A.P., and Keyser-Marcus, L. (2015). Prevalence of mental health conditions after military blast exposure, their co-occurrence, and their relation to mild traumatic brain injury. Brain Inj. 29, 1581-1588.

51. Tschiffely, A.E., Ahlers, S.T., and Norris, J.N. (2015). Examining the relationship between blast-induced mild traumatic brain injury and posttraumatic stress-related traits. J. Neurosci. Res. 93, 1769-1777.

52. Kamnaksh, A., Kwon, S.K., Kovesdi, E., Ahmed, F., Barry, E.S., Grunberg, N.E., Long, J., and Agoston, D. (2012). Neurobehavioral, cellular, and molecular consequences of single and multiple mild blast exposure. Electrophoresis 33, 3680-3692.

53. Rubovitch, V., Pharayra, A., Har-Even, M., Dvir, O., Mattson, M.P., and Pick, C.G. (2019). Dietary energy restriction ameliorates cognitive impairment in a mouse model of traumatic brain injury. J. Mol. Neurosci. 67, 613-621.

54. Rich, N.J., Van Landingham, J.W., Figueiroa, S., Seth, R., Corniola, R.S., and Levenson, C.W. (2010). Chronic caloric restriction reduces tissue damage and improves spatial memory in a rat model of traumatic brain injury. J. Neurosci. Res. 88, 2933-2939.

Address correspondence to: Peethambaran Arun, PhD Blast-Induced Neurotrauma Branch Walter Reed Army Institute of Research 503 Robert Grant Avenue Silver Spring, MD 20910

E-mail: peethambaran.arun.ctr@mail.mil 